\title{
PERFILES DE ESCRITORES: CALIDAD Y RECURSOS DE LENGUAJE ACADÉMICO EN ENSAYOS ESCRITOS POR ESTUDIANTES DE $8^{\circ}$ BÁSICO
}

\section{WRITER'S PROFILES: QUALITY AND ACADEMIC LANGUAGE RESOURCES IN ESSAYS WRITTEN BY 8TH-GRADERS}

\author{
$*$

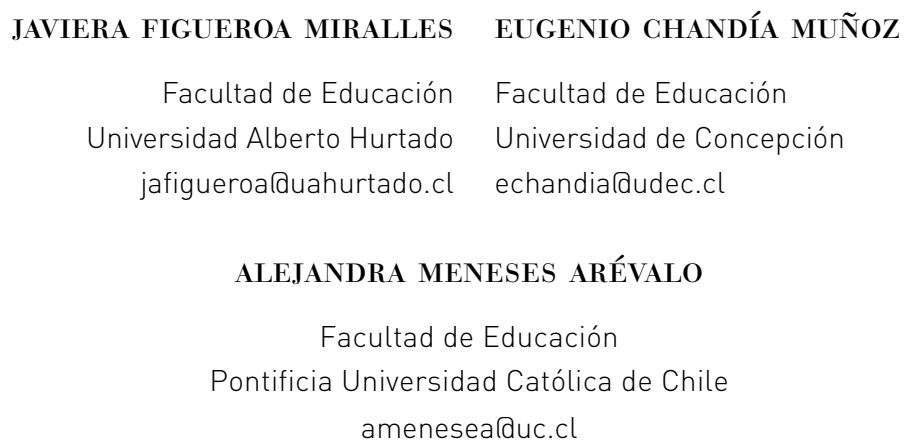

La argumentación es una habilidad que favorece el razonamiento lógico, así como la participación social y la construcción de la ciudadanía, por lo que se ha convertido en un objeto de aprendizaje relevante para la escuela; sin embargo, la construcción de argumentaciones escritas para estudiantes escolares conlleva altas demandas cognitivas y lingüísticas. Pocos estudios se han realizado sobre tipos de escritores escolares considerando estas dimensiones. El propósito de esta investigación es explorar cuáles son los perfiles de escritores con respecto a la calidad de las producciones argumentativas, así como establecer relaciones de estos perfiles con los recursos de lenguaje académico (RLA) que los estudiantes despliegan. 126 estudiantes chilenos de $8^{\circ}$ básico de tres establecimientos educacionales escribieron un ensayo sobre el uso del tablet en la sala de clases. Para evaluar la calidad, se utilizó una rúbrica que determina el dominio de las habilidades argumentativas de los escritores. Además, se codificaron recursos de lenguaje académico-vocabulario académico, nominalización, marcadores discursivos, marcadores 
106 - Cogency, Journal of reasoning and argumentation

epistémicos y deóntico- usando CLAN. Para determinar los perfiles, se realizó un análisis de clases latentes (ACL). Un 69\% de los estudiantes se clasificaron en el perfil denominado emergente. El resto de los estudiantes pertenece al segundo perfil, denominado en desarrollo. En estos dos perfiles, se analizó el uso de RLA. En el perfil emergente, se observan dos clases de estudiantes que se diferencian sustancialmente en el uso (por presencia y/o ausencia) de nominalizaciones y vocabulario académico transdisciplinar. El perfil en desarrollo también presenta dos clases: la con mayor frecuencia con un uso homogéneo de RLA, mientras que la otra se caracteriza por el uso de vocabulario académico transdisciplinar, nominalizaciones y marcadores deónticos. Se concluye que los estudiantes necesitan más oportunidades para aprender a construir argumentaciones y desplegar recursos de lenguaje académico con el objetivo de potenciar sus puntos de vista.

Palabras clave: perfiles de escritores, argumentación escrita, lenguaje académico, análisis de clases latentes.

Argumentation is a skill that promotes logical reasoning, as well as social participation and the development of citizenship. Therefore, it has become a relevant teaching tool in schools. For students, however, producing written arguments demands both high levels of cognition and linguistic skills. Few studies exist that have focused on students with these attributes. The purpose of this research is to explore the profiles of student writers with respect to the quality of their written arguments, as well as to establish the link between these profiles and students' academic language resources (ALR). One hundred and twenty-six Chilean 8th graders from three schools were assigned an essay on the use of tablets in the classroom. The quality of their essays was assessed using a rubric to determine the mastery of their argumentative skills. In addition, academic language resources -academic vocabulary, nominalization, discursive markers, epistemic and deontic markers- were coded using CLAN. An analysis of latent classes (ACL) determined the profiles. A total of 69\% of the students displayed attributes of the so-called "emergent" profile. The remainder belonged to the second profile, called "developing." In these two categories, the use of ALR was analyzed. In the "emergent" profile, there are two classes of students who differ substantially in the use of nominalizations (by presence and/or absence) and cross-disciplinary academic vocabulary. The "emerging" profile also contains two types of students: some with a more homogeneous use of ALR, while others use cross-disciplinary academic vocabulary, nominalizations and deontic markers. The study concludes that students need more opportunities to learn how to build arguments and to deploy academic language resources in order to strengthen their points of view.

Key words: writers profiles, written argumentation, academic language, latent class model. 


\section{Introducción ${ }^{1}$}

La argumentación ha sido objeto de atención en educación, pues favorece el desarrollo del razonamiento lógico, habilidad recurrente por propiciar en el contexto escolar (Kuhn, Hemberger \& Khait, 2016; Larraín \& Singer, 2019; Villarroel, Garcia-Mila, Felton \& Miralda- Banda, 2019), pues no hay duda de que es una habilidad necesaria para la participación social y la construcción de ciudadanía, sobre todo en contextos de crisis sociales. A su vez, la argumentación es una habilidad clave para el aprendizaje (Kuhn et al., 2016; Larraín, Howe \& Cerda, 2014a; Villarroel et al., 2019). En los últimos años, la argumentación se ha estudiado como un promotor del aprendizaje de la ciencia (Larraín et al., 2014a), de la lengua (Marinkovich, 2007; Poblete, 2005), de la matemática (Solar, 2018) y de la historia (Henríquez, Carmona, Quinteros \& Garrido, 2018) y forma parte de los géneros escolares (Rose \& Martin, 2012). De hecho, en Chile se ha constituido como un requisito a nivel curricular (MINEDUC, 2015), pues es una habilidad transversal a las disciplinas (Taylor, Lawrence, Connor \& Snow, 2019).

La argumentación tiene como propósito persuadir, dado que un autor reflexiona en torno a un tema controversial y utiliza estrategias para conseguir la adhesión de una audiencia (Álvarez, 2001; Bronckart, 2004) y también se utiliza transversalmente para la deliberación y la construcción de ciudadanía (Larraín et al., 2014b). Pese a que en la literatura se ha discutido si hay o no diferencias entre la modalidad oral y la escrita en la argumentación (Larraín et al., 2014b), la escritura históricamente se ha considerado como un importante medio epistémico para el aprendizaje (Andueza, 2016; Emig, 1977; Miras, 2000) y, por tanto, la escritura argumentativa se convierte en una habilidad importante de desarrollar durante la escolaridad (Kuhn et al., 2016). Sin embargo, tanto la demanda cognitiva como lingüística de la argumentación es sumamente alta para los y las estudiantes en contexto escolar (Taylor et al., 2019), lo que muchas veces se ve reflejado en las diversas dificultades que experimentan respecto al rendimiento escrito en este tipo de tareas (Benítez \& Velásquez, 2000; Figueroa, Meneses \& Chandía, 2019; Parodi, 2000; Poblete, 2005; Sotomayor et al., 2016). En efecto, este tipo de tareas representa un desafío avanzado de literacidad (Taylor et al., 2019), más aún cuando las oportunidades de argumentar e interactuar son muchas veces escasas en el aula escolar (Medina, Valdivia, Gaete-Moscoso \& Galdames, 2015; Galdames, Medina, San Martín, Gaete \& Valdivia, 2011).

1 Este estudio es la continuación de la tesis doctoral de Javiera Figueroa Miralles (2018) que se ha publicado como compendio de artículos, por lo que se hace referencia a ellos en las distintas secciones de este número 
Ahora bien, las demandas cognitivas de la argumentación escrita son crecientes en la medida que se avanza en los niveles escolares. En efecto, las habilidades argumentativas escritas son particularmente exigentes porque la audiencia es virtual y el escritor no solo debe posicionarse frente al tema, sino que también debe contemplar en su discurso contraargumentos que demuestren otras posturas alternativas e incluso debe lograr rebatirlas (Larraín et al., 2014b; Taylor et al., 2019). Estas características de la escritura argumentativa conllevan enormes dificultades para los adolescentes, pues lo más común es que justifiquen su tesis, pero sin considerar la posición de su oponente (Kuhn et al., 2016), menos aún lograr la formulación de contraargumentos (Figueroa et al., 2019; Glassner \& Schwarz, 2005; Larraín et al., 2014b). Menos estudios se han detenido a indagar sobre la calidad de argumentaciones producidas por estudiantes escolares en español.

Larraín y Singer (2019) caracterizan las habilidades argumentativas de estudiantes chilenos de $4^{\circ}$ básico con el fin de contribuir en la descripción de estas habilidades en educación básica, ya que la mayoría de los estudios se concentran en los años iniciales de escolaridad o bien en la educación superior. En este estudio, las habilidades de argumentación son evaluadas a través de una prueba escrita que mide estas habilidades a través de preguntas abiertas breves. Los resultados muestran que un gran porcentaje de estudiantes de $4^{\circ}$ básico son capaces de tomar una postura (75\%), mientras que un porcentaje más bajo puede formular argumentos para apoyar su punto de vista $(58 \%)$ y un porcentaje aún menor anticipar una posición alternativa (30\%). Si bien este estudio ofrece información pedagógicamente relevante para la enseñanza de la argumentación, no explora cómo estas habilidades se ponen en acto en la producción de una argumentación escrita. En inglés, Olinghouse y Wilson (2013) estudiaron la calidad de narraciones, argumentaciones y explicaciones producidas por estudiantes de $5^{\circ}$ grado y su relación con distintas mediciones de vocabulario. En término de la calidad de la escritura, los estudiantes tuvieron un desempeño mayor en narraciones que en argumentaciones y explicaciones; sin embargo, no se encontraron diferencias significativas entre la calidad de las narraciones y argumentaciones, pero sí entre estas y las explicaciones.

En español, Sotomayor et al. (2016) analizaron también narraciones, argumentaciones y explicaciones producidas por estudiantes chilenos de $4^{\circ}$ básico y encontraron que en términos de calidad global, los estudiantes escribieron narraciones más logradas que las argumentaciones y explicaciones. Figueroa, Meneses y Chandía (2019) analizaron argumentaciones y explicaciones producidas por estudiantes de $8^{\circ}$ básico; si bien no encontraron diferencias significativas entre estas, más del $60 \%$ de los estudiantes se ubican en los niveles de logro más bajos. Por lo tanto, resulta 
de particular interés profundizar en la calidad de las argumentaciones producidas por estudiantes, contemplando la diversidad de desempeños al interior de un grupo de estudiantes de un mismo nivel educativo de educación básica, pues un análisis en profundidad entregará información relevante para comprender los tipos de enseñanza que se requiere para que todos los estudiantes pueden avanzar en esta actividad verbal fundamental para el aprendizaje y la formación ciudadana.

\section{Recursos productivos de lenguaje académico}

Las habilidades de lenguaje se siguen desarrollando durante toda la escolaridad en relación con la diversidad de contextos comunicativos (Berman \& Ravid, 2009). Las exigencias de los contextos académicos son cada vez más desafiantes y dependen en gran medida de las exigencias de las tareas y géneros discursivos nuevos a los que se exponen los estudiantes para aprender a través de las disciplinas escolares (Figueroa, Meneses \& Chandía, 2018; Snow \& Uccelli, 2009). En estos contextos, es fundamental la aparición de la voz de los estudiantes, pues es la agencia de los sujetos la que se posiciona frente a los temas (Zavala, 2011), negociando formas y significados en la construcción de su escritura. En efecto, las estrategias retóricas siguen siendo los desafíos más difíciles de sortear en los escolares chilenos (Figueroa et al., 2019; Larraín \& Singer, 2019). De ahí que sea relevante explorar cómo los y las estudiantes utilizan y despliegan el lenguaje, pues en la medida que se apropian de una diversidad de recursos discursivos y lingüísticos, sus escritos se vuelven más precisos y con mayores probabilidades de impactar favorablemente en sus audiencias virtuales.

En el desarrollo de argumentaciones escritas, los estudiantes ponen en funcionamiento recursos discursivos y léxico-gramaticales propios del lenguaje académico para posicionarse sobre un tema y desplegar argumentos pertinentes para convencer a la audiencia. En efecto, escribir argumentaciones no solo implica poner en juego las habilidades de razonamiento lógico, sino también una serie de habilidades de lenguaje, indispensables para formular argumentaciones que acierten y persuadan a la audiencia.

El lenguaje académico se ha definido como aquel uso del lenguaje propio de la escuela (Cummins, 1981; Schleppegrell, 2004), pues es utilizado frecuentemente en los contextos de aprendizaje, los textos escolares y las evaluaciones a través de las disciplinas (Grøver, Uccelli, Rowe \& Lieven, 2019; Uccelli \& Meneses, 2015). Este registro incluye vocabulario abstracto, oraciones complejas y estructuras demarcadas para presentar información (Snow \& Uccelli, 2009), recursos infrecuentes en el lenguaje coloquial. El constructo de lenguaje académico ofrece una entrada para enfrentar los desafíos comunicativos de la escuela, desde un marco pragmático y funcional (Phillips Galloway, Stude \& Uccelli, 2015; Uccelli, 2019). Actualmente, se 
ha definido y operacionalizado dicho constructo de lenguaje académico como una constelación de habilidades léxicas, gramaticales y discursivas que son requeridas en el lenguaje de la escuela, las que han sido denominadas como habilidades clave de lenguaje académico (Uccelli, Barr et al., 2015; Uccelli, Phillips Galloway et al., 2015).

La investigación reciente ha demostrado que las habilidades clave de lenguaje -medidas como lenguaje académico receptivo- predicen la comprensión de lectura tanto en inglés (Uccelli, Barr et al., 2015; Uccelli, Phillips Galloway et al., 2015) como en español (Meneses, Uccelli, Santelices, Ruiz, Acevedo \& Figueroa, 2018), además de predecir la calidad de la escritura de explicaciones y argumentaciones (Figueroa et al., 2018). Ahora bien, los últimos estudios han avanzado en la indagación respecto a los recursos de lenguaje académico productivos que los estudiantes despliegan en sus tareas de escritura. En estos, se ha encontrado que más allá del nivel socioeconómico del colegio de los estudiantes, de la extensión de los textos y de las habilidades receptivas de lenguaje académico, el uso de nominalizaciones, la presencia de vocabulario académico temático y una postura involucrada contribuyen significativamente en la calidad de argumentaciones escritas por estudiantes de $8^{\circ}$ básico (Figueroa, Meneses, Hugo \& Chandía, en revisión); en cambio, en la calidad de explicaciones además de las variables anteriores, contribuye también el vocabulario académico transdisciplinar mientras que la postura involucrada no es un predictor para este género discursivo.

En español, se ha explorado el efecto de la enseñanza integrada o no de recursos de lenguaje académico en la producción de explicaciones científicas escritas por estudiantes de $4^{\circ}$ básico (Meneses, Hugo, Montenegro, Ruiz \& Valenzuela, 2018). En este estudio, se analizaron las explicaciones iniciales y finales de 179 estudiantes según el uso de vocabulario académico transdisciplinar, vocabulario académico científico, vocabulario metalingüístico, nominalizaciones, nexos causales, conectores, marcador de fuente, marcador epistémico y postura enunciativa involucrada. De este estudio se concluyó que si bien en ambas propuestas de enseñanza los estudiantes avanzaron en el uso de un número relevante de recursos de lenguaje académico, en la unidad en que se integra la enseñanza de los recursos con el aprendizaje del género discursivo, los estudiantes avanzaron significativamente en un mayor número de recursos, sobre todo en vocabulario académico transdisciplinar, nominalizaciones, conectores y marcador de fuente. En inglés, Phillips Galloway, Qin, Uccelli y Barr (2020) exploraron las contribuciones del lenguaje académico receptivo y productivo en la calidad de resúmenes de ciencias de estudiantes estadounidenses entre $4^{\circ} \mathrm{y}$ $7^{\circ}$ grados. Los resultados muestran que las habilidades receptivas de lenguaje académico, la comprensión del texto fuente, la extensión de los textos y los recursos 
productivos del lenguaje académico -en particular, el vocabulario académico transdisciplinar- predicen la calidad de los resúmenes.

Por lo tanto, no se han realizado estudios en español que consideren la relación entre el uso de variados recursos de lenguaje académico en la producción de argumentaciones según distintos perfiles de escritores. La investigación sobre escritura académica ha explorado algunos recursos de lenguaje académico usados con frecuencia en géneros escolares tales como uso de nominalizaciones (Ávalos, Gómez, Gort \& Secada, 2017; Chamorro, Barletta, \& Mizuno, 2013), uso de vocabulario académico (Olinghouse \& Wilson, 2013) y metalingüístico (Chen \& Myhill, 2016), así como el uso de marcadores discursivos (Concha \& Paratore, 2011; McNamara, Crossley \& McCarthy, 2010), marcadores epistémicos y deónticos (Uccelli, Dobbs, \& Scott, 2013). Sin embargo, poco sabemos sobre qué recursos productivos de lenguaje académico utilizan distintos escritores para posicionarse frente a un tema y desarrollar argumentos, considerando la calidad de las argumentaciones que producen.

Estudios de esta naturaleza permitirían comprender el aporte de estos recursos de lenguaje académico al desarrollo de la calidad argumentativa de los textos escritos. Por lo tanto, esta investigación explora la relación entre las habilidades de razonamiento implicadas en la argumentación escrita y las habilidades de lenguaje académico que los estudiantes despliegan en sus producciones, considerando la alta demanda cognitiva de estas dimensiones. Comprender esta relación entre perfiles de escritores según la calidad de la argumentación y los recursos de lenguaje académico usados, permitirá contar con evidencia pedagógicamente relevante para orientar las enseñanza de las argumentaciones escritas no solo considerando las demandas específicas de la tarea de escritura, sino también la diversidad de desempeños de los escritores con el fin de poder ofrecer andamiajes más específicos que permitan que los y las estudiantes puedan cumplir con los propósitos comunicativos de este género.

\section{Perfiles de escritores}

Estudios recientes han descrito distintas maneras de caracterizar a los escritores a través de perfiles que logran destacar diversos fenómenos (Crossley, Roscoe \& McNamara, 2014; Friginal, Li \& Weigle, 2014; Myhill, 2009; Waes \& Schellens, 2003). Por ejemplo, tanto Waes y Schellens (2003) como Myhill (2009) han caracterizado cómo los estudiantes abordan el proceso de escritura. Myhill (2009) encontró cinco perfiles respecto a cómo los estudiantes (de 14 y 16 años) abordaban el proceso de escritura. Los resultados de estos cinco perfiles son: brief pausers (aquellos estudiantes 
que tienen ráfagas de escritura con pausas breves); flow writers (quienes escriben sin parar, por lo general son lo que terminan primero); sustained pausers (se caracterizan por largas pausas y episodios breves de escritura); rapid switchers (caracterizados como aquellos escritores que tienen ráfagas cortas frecuentes tanto de pausa como de actividad de escritura); stop starters (se caracterizan por ráfagas más largas de pausa y escritura). Ahora bien, lo más interesante de estos hallazgos es que se encontró una fuerte correlación entre estos perfiles y las reflexiones de los estudiantes sobre sus procesos de escritura, demostrando una gran reflexión metalingüística sobre los procesos de composición.

Crossley et al. (2014) han descrito las distintas maneras de escribir exitosamente un ensayo, mediante distintas estrategias lingüísticas y discursivas en estudiantes de $9^{\circ}, 11^{\circ}$ y primer año de universidad. Los autores encontraron cuatro perfiles. El primero es descrito como acción y representación, pues se caracteriza por una superposición de verbos por sobre las otras palabras de contenido, con uso frecuente de estrategias retóricas como la amplificación y las expresiones de elusión de compromiso. El segundo perfil fue identificado como académico, pues destaca por su alta incidencia de nominalizaciones, vocabulario académico, mayor complejidad en la construcción de las oraciones, más presencia de estrategias retóricas y menos marcadores discursivos, entre otras características. El tercer perfil fue denominado como accesible, en el que se destaca el uso variado de conectores, superposición de argumentos y palabras de contenidos, pocas oraciones y párrafos cortos, con baja diversidad léxica y poco uso de vocabulario más sofisticado. El último perfil es caracterizado como de naturaleza léxica, pues su distinción principal es su gran variedad léxica, palabras más sofisticadas, más frases nominales, más pronombres en primera persona y una mayor superposición entre las instrucciones y el ensayo. Si bien estos hallazgos son importantes, estos corresponden a escritores escolares de lengua inglesa. En consecuencia, aún falta indagar qué sucede con los estudiantes de habla hispana. El estudio de perfiles de escritores entrega información específica sobre el desempeño de subgrupos de estudiantes por lo que resulta de interés pedagógico para el diseño de intervenciones que permitan andamiar el aprendizaje de la escritura de géneros demandantes.

En este contexto, el propósito de esta investigación es explorar cuáles son los perfiles de escritores respecto a la calidad de las producciones argumentativas, así como establecer relaciones de estos perfiles con los recursos de lenguaje académico (RLA) que los estudiantes despliegan en el desarrollo de argumentaciones escritas. Para tal efecto, primero se describen y ejemplifican los perfiles respecto a la calidad de las argumentaciones escritas y, posteriormente, se profundiza en cada perfil respecto al uso de recursos del lenguaje académico desplegados en cada grupo. 


\section{Método}

\subsection{Participantes}

La muestra estuvo conformada por 126 estudiantes de $8^{\circ}$ básico, distribuidos en tres establecimientos educacionales ubicados en la Región Metropolitana en Santiago de Chile. Para asegurar la heterogeneidad de la muestra, se seleccionaron tres establecimientos correspondientes a los grupos socioeconómicos (GSE) alto, medio y medio-bajo (Agencia de Calidad de la Educación, 2015), debido a la alta segregación del sistema educativo chileno (Valenzuela, Bellei \& De Los Ríos, 2014). Por tanto, el muestreo fue no probabilístico intencional a partir de su GSE y tipo de dependen$\mathrm{cia}^{2}$. Los estudiantes tienen entre 13 y 14 años de edad. En la tabla 1 se observa la distribución de estudiantes por género ( $49 \%$ mujeres y $51 \%$ hombres) y por tipo de establecimiento educativo.

Tabla 1.

Distribución de los participantes

\begin{tabular}{|l|c|c|c|}
\hline \multirow{2}{*}{ GSE } & \multicolumn{3}{|c|}{$8^{0}$ básico } \\
\cline { 2 - 4 } & Femenino & Masculino & 51 \\
\hline Alto & 27 & 22 & 40 \\
\hline Medio & 18 & 18 & 35 \\
\hline Medio Bajo & 17 & 64 & 126 \\
\hline Total & 62 & 22 & 5 \\
\hline
\end{tabular}

\subsection{Procedimientos}

La tarea de escritura se administró grupalmente en la que se destinaron 30 minutos para su desarrollo y fue escrita a mano por los estudiantes. Estas fueron aplicadas por la investigadora principal. Se recolectaron los consentimientos de los padres y los asentimientos de los estudiantes, quienes aceptaron participar voluntariamente del estudio. Aquellos estudiantes que decidieron no participar permanecieron en la sala realizando otra actividad de su interés.

\footnotetext{
2 Si bien el objetivo de este estudio no es comparar por GSE, otros estudios sí han comparado los GSE de esta muestra según otras variables de interés como el desempeño en cada una de las dimensiones de la rúbrica (Figueroa et al., 2019) o la predicción de la calidad de la escritura (Figueroa et al., 2018).
} 


\subsection{Instrumentos}

\subsubsection{Tarea de escritura}

Cada estudiante realizó una tarea argumentativa a partir del tema "El uso de tablets en la sala de clases". Se les solicitó escribir un ensayo sobre una situación ficticia a partir de un conflicto en un contexto escolar, respecto al uso del tablet como medio para el aprendizaje. Las instrucciones fueron explícitas sobre los que debían escribir: "Escribe un ensayo sobre los usos del tablet en la sala de clases para una revista escolar sobre tecnología. En este ensayo debes dar un punto de vista y argumentos para convencer a los lectores de tu postura. Los siguientes son elementos que debe tener tu ensayo: una introducción, un punto de vista sobre esta situación, argumentos que apoyen tu postura, un argumento que se contraponga al tuyo (por ejemplo, las razones que tiene el director para no permitir su uso) y una conclusión" (Figueroa, Chandía \& Meneses, 2018).

\subsubsection{Evaluación de la escritura}

Para determinar la calidad de los ensayos, se llevó a cabo un proceso de adaptación de una rúbrica (Figueroa et al., 2018), utilizada en NAEP. Las dimensiones que se evaluaron fueron posición (POS), entendida como la capacidad de tomar una postura frente a un tema; ideas o argumentos (IDEA), que evalúa el despliegue y elaboración de las ideas que realiza el escritor para defender su punto de vista; contraargumento (CONTRA), como aquella actividad retórica utilizada para resaltar la propia postura; y organización (ORGA), cuando la información está estructurada según las demandas de la tarea. El puntaje total es de 16 puntos, el que se obtuvo mediante la suma total de cada dimensión (el puntaje máximo por dimensión es de 4). Es importante destacar que para esta rúbrica la operacionalización de la calidad de la escritura no incorporó dimensiones del lenguaje (como vocabulario y conexión) y se focalizó más bien en dimensiones discursivas (Figueroa et al., 2018). Si bien la rúbrica considera cuatro niveles, en la tabla 2 se presentan las descripciones para los niveles logrado y no logrado para tener una comprensión más acabada de lo que se evalúa. 
Tabla 2.

Nivel 3 y 1 de la rúbrica (Figueroa et al., 2018)

\begin{tabular}{|c|c|c|}
\hline $\begin{array}{l}\text { Nivel } \\
\text { Dimensión }\end{array}$ & $\begin{array}{l}\text { Nivel } 3 \\
\text { Logrado }\end{array}$ & $\begin{array}{l}\text { Nivel } 1 \\
\text { No logrado }\end{array}$ \\
\hline $\begin{array}{l}\text { Posición } \\
\text { (POS) }\end{array}$ & $\begin{array}{l}\text { El texto formula una posición sobre el uso } \\
\text { del Tablet en la sala de clases. } \\
\text { El texto demuestra cierta comprensión de } \\
\text { otras perspectivas, aunque no las incorpora } \\
\text { para dar mayor profundidad al tema. } \\
\text { La otra perspectiva está presente, pero no } \\
\text { nominada; es decir, no se explicita el sujeto } \\
\text { de esa otra posición. Por ejemplo: "Algunos } \\
\text { piensan", "todos saben", “otros creen”. }\end{array}$ & $\begin{array}{l}\text { El texto no indica una posición sobre el } \\
\text { uso del Tablet en la sala de clases, pues } \\
\text { entrega ventajas y desventajas sin expli- } \\
\text { citar una posición, o bien realiza una des- } \\
\text { cripción de la situación o una narración. } 0 \\
\text { bien, hay posición contradictoria (parte con } \\
\text { A y termina con B). El texto no incorpora } \\
\text { otra perspectiva. }\end{array}$ \\
\hline $\begin{array}{l}\text { Ideas/ } \\
\text { Argumentos } \\
\text { (IDEA) }\end{array}$ & $\begin{array}{l}\text { El texto proporciona razones persuasivas. } \\
\text { Hay por lo menos dos razones o argumentos } \\
\text { para respaldar la postura, pero están media- } \\
\text { namente desarrollados. }\end{array}$ & $\begin{array}{l}\text { El texto proporciona razones superficiales } \\
\text { (prejuicios y/o descalificaciones). } \\
\text { Hay solo un argumento para respaldar } \\
\text { la postura y está poco desarrollado o sin } \\
\text { desarrollo. }\end{array}$ \\
\hline $\begin{array}{l}\text { Contraargumento } \\
\text { (CONT) }\end{array}$ & $\begin{array}{l}\text { El texto menciona un contraargumento me- } \\
\text { dianamente desarrollado. }\end{array}$ & El texto no menciona un contraargumento. \\
\hline $\begin{array}{l}\text { Organización } \\
\text { (ORGA) }\end{array}$ & $\begin{array}{l}\text { El texto demuestra una organización lin- } \\
\text { troducción, desarrollo y conclusión), pues } \\
\text { la mayoría de las ideas están lógicamente } \\
\text { agrupadas y reflejan el enfoque del escritor. } \\
\text { Por lo general, los párrafos se desarrollan en } \\
\text { torno al uso del Tablet en la sala de clases. }\end{array}$ & $\begin{array}{l}\text { El texto no muestra una organización. } \\
\text { Las ideas son agrupadas de forma ilógica } \\
\text { (listado de ideas o ideas inconexas en un } \\
\text { solo gran párrafo). } \\
\text { No hay construcción de párrafos. }\end{array}$ \\
\hline
\end{tabular}

\subsubsection{Codificación de recursos de lenguaje académico (RLA)}

Todos los recursos de lenguaje académico que se consideraron en esta investigación fueron seleccionados de acuerdo con una revisión del total de palabras producidas por los estudiantes. De este modo, se creó un listado de palabras a partir del corpus total (1824 palabras distintas de un total de 13948) y luego se clasificaron los RLA. Los listados de recursos fueron desambiguados y revisados por tres expertos. Además, se cotejaron con una búsqueda de los corpus disponibles de textos académicos en español (Corpus del Español del Siglo XXI: Real Academia Española, 2016). Esto permitió recopilar pruebas de la prevalencia de estas palabras en el discurso académico en español. En la tabla 3, se sistematizan los recursos de lenguaje académico codificados.

\subsection{Análisis de datos}

Para el análisis de datos todos los textos se transcribieron con el objetivo de evitar el sesgo a partir de la caligrafía o la ortografía. La calidad de la escritura fue revisada por dos correctores con experiencia en revisión de muestras de escritura y fueron capacitados para realizar la evaluación de los textos. Además, se doble codificó un 20\% de la muestra y se calcularon los índices Kappa de Cohen para cada dimensión, que demuestran la confiabilidad de la codificación $(\mathrm{POS}=.93 ; \mathrm{IDEA}=.83 ; \mathrm{CONT}=.71 ; \mathrm{ORGA}=.91)$. 
Tabla 3.

Recursos de lenguaje académico (RLA) codificados

\begin{tabular}{|c|c|c|c|}
\hline \multicolumn{2}{|c|}{ Recurso de lenguaje académico RLA } & Definición & Ejemplo \\
\hline \multirow{3}{*}{$\begin{array}{l}\text { Vocabulario } \\
\text { académico }\end{array}$} & $\begin{array}{l}\text { Transdiscipli- } \\
\text { nar }\end{array}$ & $\begin{array}{l}\text { Palabras de naturaleza abstracta y poco frecuen- } \\
\text { tes en el lenguaje cotidiano, en las que se incluye } \\
\text { el vocabulario utilizado a través de las disciplinas } \\
\text { para explicar conceptos (Coxhead, 2000). }\end{array}$ & $\begin{array}{l}\text { impedir } \\
\text { sistema }\end{array}$ \\
\hline & Temático & $\begin{array}{l}\text { Palabras utilizadas en las instrucciones de la ta- } \\
\text { rea y que son utilizadas por los estudiantes en el } \\
\text { texto producido (Gómez et al., 2016). }\end{array}$ & $\begin{array}{l}\text { tablet } \\
\text { aplicaciones }\end{array}$ \\
\hline & Metalingüístico & $\begin{array}{l}\text { Palabras utilizadas para referirse a los géneros } \\
\text { discursivos, procesos de escritura o sobre el len- } \\
\text { guaje (Myhill \& Jones, 2015). }\end{array}$ & $\begin{array}{l}\text { ensayo } \\
\text { introducción }\end{array}$ \\
\hline \multicolumn{2}{|c|}{ Nominalización } & $\begin{array}{l}\text { Sustantivos que derivan de una transformación } \\
\text { gramatical de un verbo o de un adjetivo (García, } \\
\text { Hall y Marín, 2005). }\end{array}$ & $\begin{array}{l}\text { comportamiento } \\
\text { determinación }\end{array}$ \\
\hline \multirow{2}{*}{$\begin{array}{l}\text { Marcador } \\
\text { discursivo }\end{array}$} & Formulativos & $\begin{array}{l}\text { Piezas lingüísticas que se utilizan como pistas } \\
\text { para organizar la información en un texto (Errázu- } \\
\text { riz, 2012; Montolío, 2001). }\end{array}$ & $\begin{array}{l}\text { en primer lugar } \\
\text { en conclusión }\end{array}$ \\
\hline & Lógicos & $\begin{array}{l}\text { Conector que establece una relación lógica entre } \\
\text { dos oraciones (Montolío, 2001). }\end{array}$ & $\begin{array}{l}\text { sin embargo } \\
\text { en cambio }\end{array}$ \\
\hline \multicolumn{2}{|c|}{ Marcador epistémico } & $\begin{array}{l}\text { Piezas lingüísticas que se utilizan para expresar } \\
\text { el grado de certeza o creencia del escritor sobre } \\
\text { una determinada proposición (Berman, 2004; Uc- } \\
\text { celli et al., 2013). }\end{array}$ & $\begin{array}{l}\text { creo } \\
\text { probablemente }\end{array}$ \\
\hline \multicolumn{2}{|c|}{ Marcador deóntico } & $\begin{array}{l}\text { Piezas lingüísticas que expresan deber (Uccelli et } \\
\text { al., 2013). }\end{array}$ & $\begin{array}{l}\text { debe } \\
\text { debería }\end{array}$ \\
\hline
\end{tabular}

Para realizar el análisis de los recursos de lenguaje académico, se utilizaron los programas de análisis de lenguaje automático de CHILDES (Child Language Data Exchange System) CHAT y CLAN (MacWhinney, 2014). Se segmentó el corpus y se revisó el total de la muestra por dos investigadores expertos. Asimismo, se construyeron los listados de palabras para los otros recursos léxicos, que fueron revisados por tres expertas y, posteriormente, se utilizó CLAN con el objetivo de calcular la frecuencia de aparición para cada recurso identificado.

Para determinar los perfiles se realizó un análisis de clases latentes (ACL). El ACL es quizás el modelo de mezcla más directo que actualmente se utiliza para identificar subgrupos de individuos mutuamente excluyentes basados en sus respuestas a las variables medidas (Clogg y Goodman, 1984). Los modelos ACL explican las relaciones entre las variables categóricas en una tabla de contingencia clasificada de forma cruzada al suponer la existencia de una clasificación no observada o latente. Esto permite clasificar a los estudiantes en función de la calidad de las argumentaciones 
escritas y de los recursos de lenguaje académico. Para determinar el número de clases latentes se han utilizado una combinación de criterios de información, el criterio de información de Akaike (Akaike, 1987) y el consistente (Nylund, Asparouhov \& Muthén, 2007), el criterio de información bayesiano (Schwartz, 1978) y el ajustado (Nylund, et al., 2007). Además de los índices de razón de probabilidad y el nivel de entropía (Celeux \& Soromenho, 1996). Todos los análisis se llevaron a cabo con paquetes del software R (R Development Core Team, 2017).

\section{Resultados}

\subsection{Perfiles en la calidad de los ensayos escritos}

Los resultados del análisis de clases latentes de la rúbrica sugirieron que los participantes formaron dos grupos que tuvieron un rendimiento diferente al desarrollar el ensayo, dado que el modelo con dos clases obtuvo los mejores ajustes $(\mathrm{BIC}=1154.03$, $\mathrm{aBIC}=1096.17, \mathrm{AIC}=1083.12, \mathrm{cAIC}=1021.43$, G2=118.82, Entropy=0.86). La probabilidad de pertenecer a la clase 1 fue igual a $69 \%$ y la probabilidad de pertenecer a la segunda clase fue del $31 \%$.

Tabla 4.

Probabilidades de presentar las dimensiones de calidad en cada clase

\begin{tabular}{|l|c|c|c|c|c|c|c|c|}
\hline \multirow{2}{*}{} & \multicolumn{4}{|c|}{ Clase 1: emergente } & \multicolumn{5}{c|}{ Clase 2: en desarrollo } \\
\cline { 2 - 9 } & \multicolumn{3}{|c|}{.69} & \multicolumn{5}{c|}{.31} \\
\cline { 2 - 9 } & Nivel 1 & Nivel 2 & Nivel 3 & Nivel 4 & Nivel 1 & Nivel 2 & Nivel 3 & Nivel 4 \\
\hline POS & .07 & .92 & .01 & .00 & .04 & .39 & .36 & .22 \\
\hline IDEA & .44 & .52 & .04 & .00 & .13 & .50 & .33 & .05 \\
\hline CONT & .70 & .20 & .10 & .00 & .33 & .34 & .17 & .17 \\
\hline ORGA & .45 & .29 & .13 & .13 & .04 & .16 & .26 & .53 \\
\hline
\end{tabular}

POS: posición; IDEA: ideas/argumentos; CONT: contraargumento; ORGA: organización

La clase 1 (ver Tabla 5), denominada como emergente, la componen estudiantes de $8^{\circ}$ básico que presentan una alta probabilidad de tener una posición débil frente al tema planteado, en la que se entregan ventajas y desventajas sobre este, pero no hay una posición clara ni se incorpora otra perspectiva. En relación a la organización del texto, estos estudiantes presentan alta probabilidad (más del 50\%) de obtener una estructura organizativa incompleta o sin una estructura que organice la información. Sobre el desarrollo de las ideas, los estudiantes de este perfil tienen mayores probabilidades de construir argumentos o razones poco desarrolladas o sin desarro- 
llar. Por último, presentan altas probabilidades de no utilizar contraargumentos o de utilizarlos de manera superficial.

Por el contrario, la clase 2, denominada como en desarrollo, la componen estudiantes de $8^{\circ}$ básico que presentan una alta probabilidad de tomar una posición clara en la elaboración de sus textos e incorporan otra perspectiva, la que se utiliza para otorgar mayor profundidad al tema. En relación a la organización del texto, estos estudiantes demuestran altas probabilidades de ordenar lógicamente sus ideas a través de una estructura organizativa y desarrollar párrafos de forma efectiva. Respecto del desarrollo de las ideas, se presentan algunos argumentos o razones, los que están medianamente desarrollados. Por último, sobre el uso de contraargumentos, los estudiantes varían en el uso de este recurso retórico, pues la mayoría lo utiliza, aunque de forma superficial e incluso en muchos casos no se utiliza. En la tabla 5, se presentan ejemplos para cada perfil identificado.

\section{Tabla 5.}

Ejemplos de perfil emergente y en desarrollo

Perfil 1: emergente

¿Está bien o está mal?

¿Estarían bien o mal? Bien porque ayudaría a los estudiantes a mejorar su aprendizaje, mal porque algunos estudiantes entraría a otras páginas 0 podría ocurrir ciberbullying, etc.

Estaría bien porque ayudaría a los alumnos a aprender más, investigar, buscar palabras que no entienden, etc.

Mal porque los estudiantes podrían hacerse Bullying o ver páginas no permitidas, etc.

No deberían sacarlas y bloquear las aplicaciones que los estudiantes usan para molestar a otros.

\section{Perfil 2: en desarrollo}

Salas de clases con tablets

En este ensayo hablaré sobre mi opinión en cuanto al uso de tablets en salas de clases de colegio.

Yo opino que no se debería usar tablets en clases ya que al tener acceso a Internet los alumnos no buscarían temas interesantes de la materia o diccionarios en línea sino que buscarían temas de su interés y/o están expuestos a todo tipo de páginas y distracciones.

Es cierto que ahora se puede bloquear el acceso a ciertas páginas o distintas acciones del tablet como descargar juegos, sacar fotos, etc, pero los alumnos encontrarán una forma de distraerse usando estos tablets.

En conclusión creo que los tablets no ayudarían al estudio en las salas de clases sino que son una distracción para los alumnos.

Tal como se demuestra en los ejemplos, el estudiante del perfil emergente no logra posicionarse frente a la controversia y más bien esboza ventajas y desventajas sobre la situación planteada, por lo que la audiencia virtual no lograría adoptar el punto de vista del escritor. En cambio, el estudiante del perfil en desarrollo se posiciona frente al tema planteado, desarrolla argumentos para respaldar su postura e incluso logra 
incorporar un contraargumento y luego rebatirlo. Además, utiliza la conclusión como una forma retórica de enfatizar al lector virtual su punto de vista.

\subsection{Perfiles de los recursos de lenguaje académico en el desarrollo de los ensayos}

En estos dos perfiles (emergente y en desarrollo) se realizó un ACL en el uso de recursos de lenguaje académico que los estudiantes desplegaron en sus ensayos. En el perfil emergente se observan dos clases con buenos ajustes $(\mathrm{BIC}=-1833.01, \mathrm{aBIC}=-$ 1911.90, AIC $=-1894.94, \mathrm{cAIC}=-1808.01, \mathrm{G} 2=972.47$, Entropy=0.78) de estudiantes que se diferencian sustancialmente en el uso de nominalizaciones y vocabulario transdisciplinar, por sobre los otros recursos analizados, tal como se observa en la distribución de probabilidades de cada recurso en la Figura 1. El primer grupo de estudiantes ( $n=56$, rojo) se caracteriza por presentar mayores probabilidades de presentar el recurso de vocabulario temático y menor probabilidad de usar los recursos de vocabulario transdisciplinar y de nominalizaciones, que son los recursos más complejos y sofisticados de la lengua. El segundo grupo de este perfil, lo componen estudiantes $(n=32$, azul) que presentan mayor probabilidad de utilizar los recursos de nominalizaciones y vocabulario transdisciplinar en la escritura de su ensayo, dejando de lado el uso preferente de vocabulario temático.

\section{Figura 1.}

Distribución de las probabilidades de presentar los RLA en los ensayos en el perfil emergente

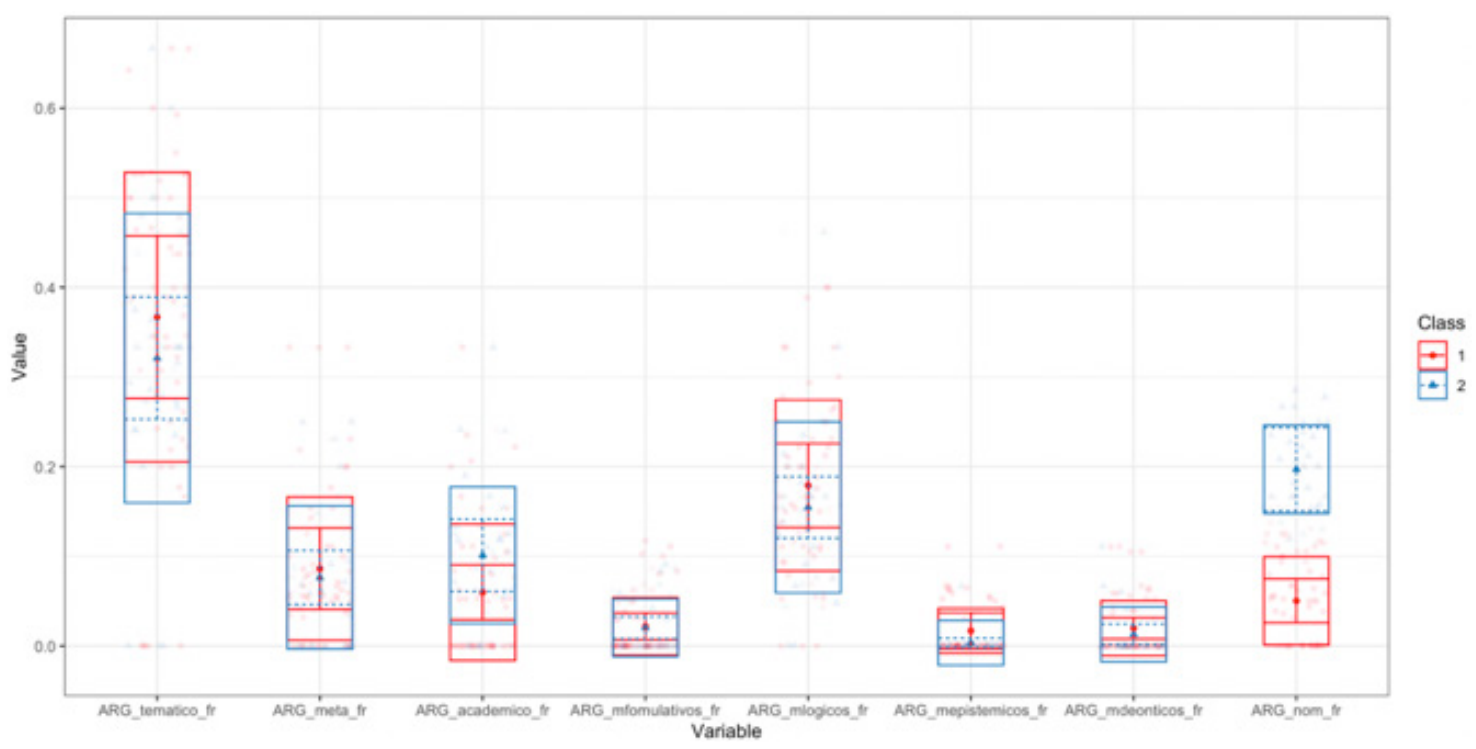

En la Tabla 6, se presentan ejemplos que permiten evidenciar la presencia y ausencia de RLA diferenciadores entre los grupos identificados para el perfil emergente. 


\section{Tabla 6.}

Ejemplos grupo 1 y 2 perfil emergente

\begin{tabular}{|c|c|}
\hline Grupo 1 & Grupo 2 \\
\hline 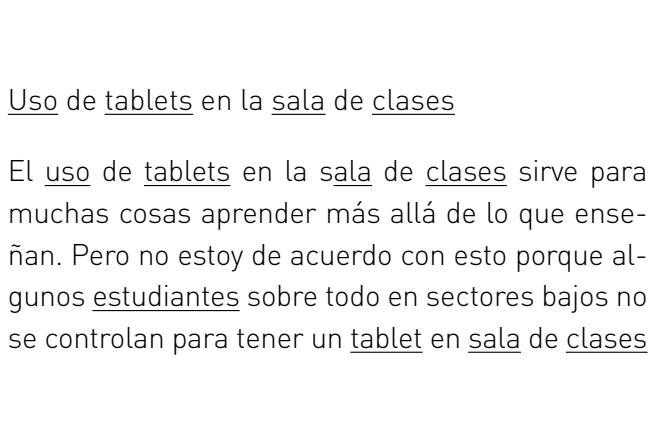 & $\begin{array}{l}\text { El uso de tablets es muy buena idea porque facilita } \\
\text { los trabajos, buscar información, etc. } \\
\text { Aunque algunos creen que es mala idea porque } \\
\text { suben cosas que hablan mal de sus compañeros. } \\
\text { Igual algunos buscan información interesante, dic- } \\
\text { cionarios online, etc los padres están preocupados } \\
\text { por la situación de los niños, y los directores no } \\
\text { están de acuerdo con el uso de tablets en la sala } \\
\text { de clases }\end{array}$ \\
\hline
\end{tabular}

Nota: vocabulario temático, vocabulario transdiciplinar, nominalizaciones

\section{Figura 2.}

Distribución de las probabilidades de presentar RLA en los ensayos en el perfil en desarrollo

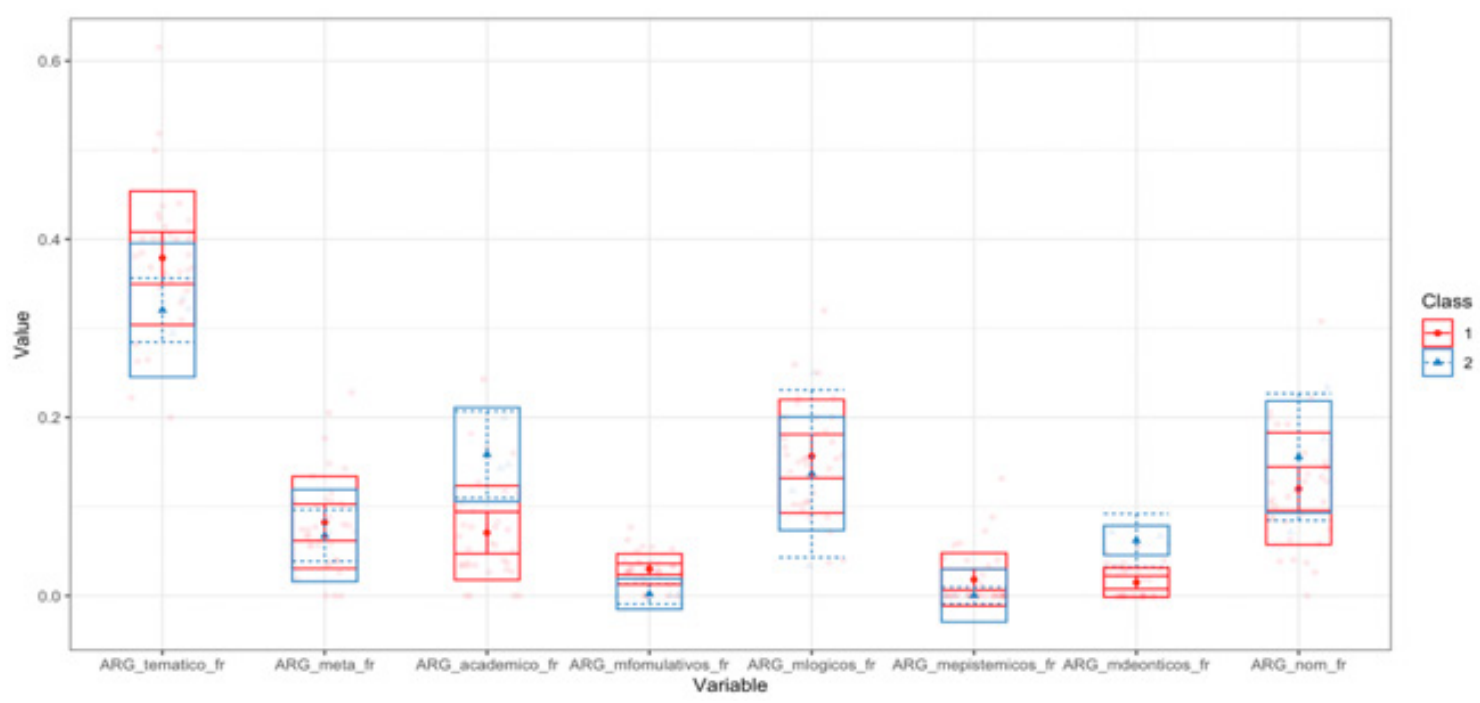

Por otra parte, el perfil en desarrollo también presenta dos clases de estudiantes con buenos índices de ajuste $(\mathrm{BIC}=-979.88, \mathrm{aBIC}=-1058.05, \mathrm{AIC}=-1020.82, \mathrm{cAIC}=$ -954.88, G2=535.41, Entropy=0.95). El primero tiene mayor frecuencia $(n=35$, rojo); sin embargo, el uso de recursos en sus textos argumentativos es más bien homogéneo con probabilidades bajas tal como se observa en la Figura 2, con excepción del recurso de vocabulario temático. Es decir, estos estudiantes cumplen con las demandas de la tarea para desarrollar la argumentación escrita, pero no presentan probabilidades de usar un lenguaje más especializado. En cambio, la segunda clase (azul), compuesta solo por tres estudiantes, se caracteriza por presentar mayores probabilidades para desplegar recursos de lenguaje académico, específicamente, 
vocabulario transdisciplinar, nominalizaciones y marcadores deónticos. Estos casos corresponden a estudiantes que logran combinar un buen desempeño argumentativo con un buen dominio y despliegue de lenguaje académico. Los ejemplos de cada grupo se encuentran en la Tabla 7.

En síntesis, del análisis de clases latentes, emergieron dos clases de perfiles de escritores: emergente y en desarrollo. El perfil de escritor emergente se caracteriza por tener una alta probabilidad de presentar sobre todo ventajas y desventajas sobre el tema, sin una construcción precisa y robusta de un posicionamiento sobre la controversia planteada. Asimismo, la argumentación presenta una organización incompleta o bien no presenta una estructura lógica que favorezca la comprensión; se introducen argumentos que no son debidamente desarrollados y no se incorporan contraargumentos o se los incorpora de manera superficial. Por su parte, en el perfil de escritor en desarrollo se encuentran estudiantes con una alta probabilidad de desarrollar una posición clara e incorporar otra perspectiva. Sus argumentaciones estructuran lógicamente las ideas y con argumentos medianamente desarrollados; asimismo no incorporan contraargumentos o los introducen de manera superficial.

Tabla 7.

Ejemplos grupo 1 y 2 perfil en desarrollo

\begin{tabular}{|c|c|}
\hline Grupo 1 & Grupo 2 \\
\hline 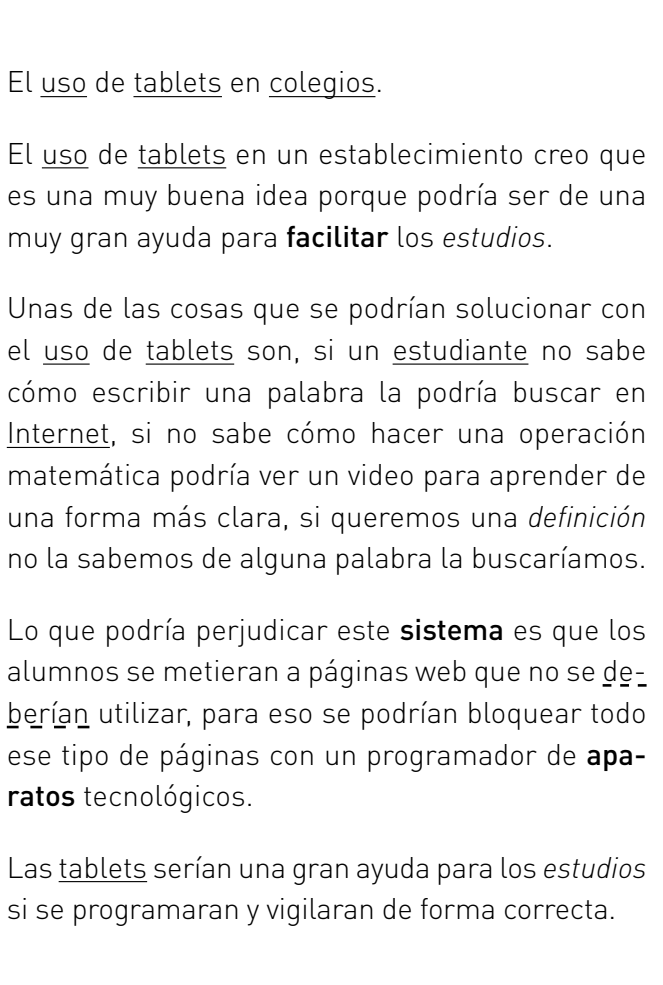 & 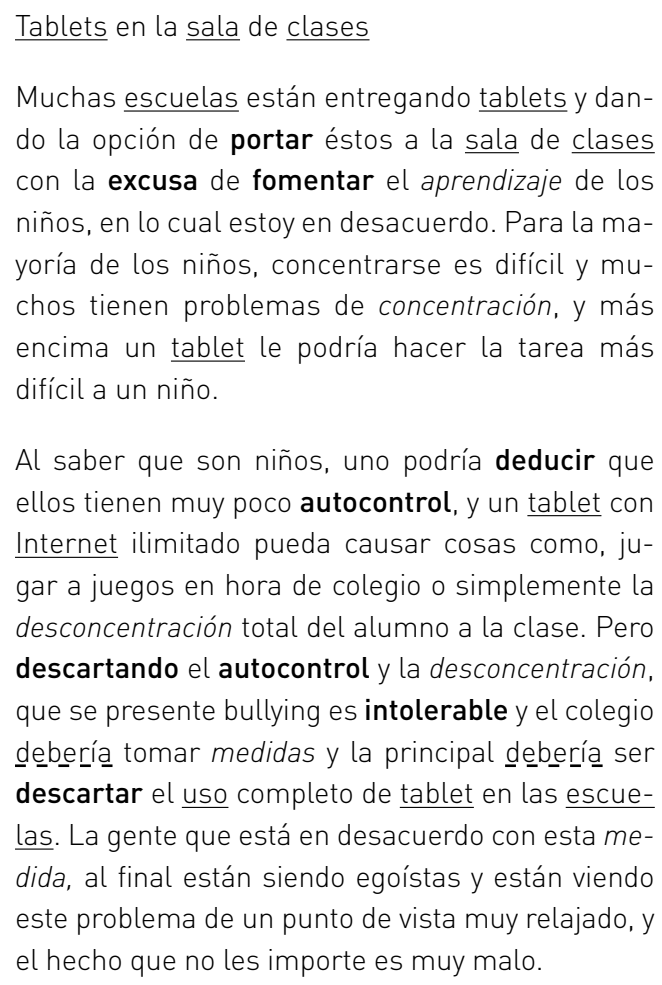 \\
\hline
\end{tabular}

Nota. vocabulario temático, vocabulario transdiciplinar, nominalizaciones, marçado_e_es__eón 
Se establecen al interior de estos perfiles escritores, subgrupos según los recursos productivos de lenguaje académico utilizados. Al interior del perfil escritor emergente, se encuentran dos subgrupos: escritores con probabilidad de utilizar vocabulario temático y escritores con probabilidad de usar nominalizaciones y vocabulario transdisciplinar. En el perfil escritor en desarrollo, también se encontraron dos subgrupos, a saber, aquellos que utilizan de manera homogénea los recursos y aquellos que despliegan en sus textos más vocabulario transdisciplinar, nominalizaciones y marcadores deónticos.

\section{Discusión}

Este artículo se ha propuesto explorar perfiles de escritores según la calidad de las argumentaciones escritas por estudiantes chilenos de $8^{\circ}$ básico, así como establecer relaciones entre estos perfiles y los usos de recursos de lenguaje académico en los ensayos producidos. Los porcentajes para la probabilidad de pertenecer al perfil emergente (69\%) o al perfil en desarrollo (31\%) demuestran que un gran número de estudiantes de $8^{\circ}$ básico no ha alcanzado aún el dominio de las habilidades argumentativas. En efecto, no se encontró un perfil de escritor óptimo; además, la cantidad de ensayos correspondientes al perfil en desarrollo es menor que aquellos que se encuentran en un perfil emergente.

Para alcanzar un nivel satisfactorio, los escritores requieren andamiajes específicos para construir argumentos desarrollados, así como enseñanza explícita para incorporar satisfactoriamente contraargumentos. Por su parte, los escritores del perfil emergente requieren de andamiajes discursivos para representarse y producir de manera adecuada las fases de la argumentación (posición, argumentos y contraargumentos), tanto como estrategias para estructurar lógicamente los ensayos. Estos resultados demuestran que las habilidades argumentativas escritas son complejas para los estudiantes escolares, pues la construcción de una argumentación de calidad no requiere solo de conocimiento del tema, sino también de un conocimiento discursivo específico para la formulación del posicionamiento y el desarrollo de argumentos y contraargumentos pertinentes que apoyen la postura planteada (Larraín et al., 2014b; Taylor et al., 2019).

Larraín y Singer (2019) describieron las habilidades argumentativas a través de preguntas específicas, encontraron que un $75 \%$ los estudiantes chilenos de $4^{\circ}$ básico toman una postura y un $58 \%$ formula argumentos para apoyar un punto de vista mientras que solo un $30 \%$ explicita una postura alternativa. Estos resultados difieren en parte de lo encontrado en este estudio, ya que un mayor número de estudiantes de $8^{\circ}$ básico se ubican en el perfil emergente, lo que quiere decir que no toman una postura de manera precisa y no logran desarrollar suficientemente sus argumentos. 
Estas diferencias podrían vincularse con el tipo de tarea para medir las habilidades argumentativas: en este estudio se evaluaron estas de manera integrada en una tarea de producción escrita. Esto podría estar indicando que la descomposición de las habilidades argumentativas para ser evaluadas en tareas o preguntas específicas puede no sólo entregar información específica de los desempeños alcanzados por los estudiantes, sino que también ser una tarea, probablemente, más fácil que la producción de un texto en que estas habilidades se ponen en acto e interactúan con el conocimiento del tema y con las elecciones lingüísticas para la construcción de la argumentación. Más investigación desde la perspectiva del desarrollo se requiere para comprender cómo progresan las habilidades argumentativas, así como explorar las relaciones entre distintas tareas para evaluarlas en términos de su complejidad.

Por otra parte, estos resultados sugieren que si bien la investigación ha destacado el papel de la argumentación en el aprendizaje escolar (Kuhn et al., 2016; Larraín \& Singer, 2019; Taylor et al., 2019), en el bajo logro de los ensayos producidos se evidencia que los estudiantes no han tenido suficientes oportunidades para aprender a construir argumentaciones tanto a nivel macro (posicionamiento y organización) como a nivel micro (argumentos y contraargumentos). Como plantea Kuhn et al. (2016), la estimación de otra perspectiva para la construcción del posicionamiento es una tarea sumamente desafiante para los adolescentes, tanto como la incorporación adecuada de contraargumentos (Glassner \& Schwarz, 2005). Por lo tanto, la representación adecuada de la tarea escrita (ensayo), la descomposición en fases discursivas, el modelaje por parte de un escritor experto y la escritura compartida son algunas de las estrategias efectivas que se han propuesto desde la pedagogía del género (Rose \& Martin, 2012) y que se podrían implementar en las aulas chilenas para promover el aprendizaje de la escritura de argumentaciones.

Sobre los perfiles de escritores, Myhill (2009) determinó cinco perfiles a través de la identificación cualitativa de patrones de comportamiento observados durante la escritura de un texto. El estudio fue conducido con 38 estudiantes británicos entre 14 y 16 años, participantes de edades cercanas a los de esta investigación. Los perfiles fueron construidos considerando las pausas que realizaban los escritores durante su proceso de escritura. El mayor porcentaje de estudiantes se ubicó en el perfil de escritores que realizan cambios rápidos entre pausas y escritura (rapid switchers, 39\%) y aquellos que tomaban pausas breves (brief pausers, 30\%). Además, la autora analizó la relación de estos perfiles con las reflexiones sobre sus procesos de escritura: los escritores que cambian rápido entre la tarea de escritura y las pausas reportan estrategias para aumentar la concentración. En cambio, los escritores que escriben sin pausas reportan no detenerse a pensar ni a planificar su escritura. El estudio reportado en 
este artículo también explora patrones de escritores adolescentes, pero se enfoca en los productos escritos en español configurando subgrupos no solo según la calidad de la argumentación, sino también según los recursos de lenguaje académico usados por los escritores.

Por lo tanto, se contribuye con evidencia en español sobre perfiles de escritores relacionando estrategias discursivas y recursos lingüísticos, información pedagógicamente relevante para apoyar a los escritores en el aprendizaje de un género discursivo complejo. Tal como en el estudio de Myhill (2009), se encuentra una variabilidad de perfiles escritores al interior del grupo lo que releva los desafíos pedagógicos para poder ofrecer oportunidades más específicas para andamiar a los distintos escritores en sus procesos de escritura de géneros escolares desafiantes. Por otra parte, los resultados empíricos de los perfiles de escritores, han sido levantados para el ensayo; más investigación se requiere para estudiar la variación de perfiles de escritores en distintos géneros discursivos. En efecto, la investigación de Figueroa et al. (en revisión) determinó que, además, del nivel socioeconómico del colegio, la extensión de los textos y las habilidades receptivas de lenguaje académico, el vocabulario temático, el uso de nominalizaciones y el vocabulario transdisciplinar eran recursos productivos del lenguaje académico que predicen la calidad de las explicaciones mientras que solo los dos primeros contribuyen en la calidad de las argumentaciones. Por lo tanto, sería relevante realizar el estudio de los perfiles de escritores en relación con los recursos del lenguaje académico en explicaciones para explorar la flexibilidad retórica de los escritores según las tareas desde una aproximación sociocultural y situada de la escritura.

Crossley et al. (2014), por su parte, levantaron perfiles de escritores a través de análisis de cluster, analizando las estrategias discursivas y lingüísticas usadas por estudiantes estadounidenses de los últimos años de escolaridad y de primer año de estudios superiores en la producción de ensayos. En sus hallazgos se proponen cuatro perfiles de escritores: acción y representación con mayor presencia de verbos y estrategias de amplificación y elusión de compromiso; académico con una presencia alta de vocabulario académico, nominalizaciones, complejidad sintáctica a nivel oracional, entre otros; accesible, con una alta incidencia de palabras de contenidos, con uso de oraciones y párrafos breves, baja diversidad léxica; y naturaleza léxica, con mayor presencia de palabras sofisticadas, diversidad léxica y expresiones en primera persona. Este estudio destaca la diversidad de recursos lingüísticos vinculados con la calidad de los ensayos considerando distintos tipos de escritores. En el estudio que se reporta en este artículo, se encontraron resultados similares a los de Crossley et al. (2014) con escritores adolescentes de habla hispana. Del análisis de recursos de 
lenguaje académico, dentro del perfil emergente, se identificó que un 36,4\% de los estudiantes usa nominalizaciones y vocabulario académico transdisciplinar en sus ensayos, mientras que un $63,6 \%$ de los estudiantes no despliega dichos recursos en las argumentaciones producidas. En el perfil en desarrollo, se encontró que un 92,1\% de los escritores utiliza una variedad de recursos de lenguaje académico de manera homogénea; en cambio, solamente un 7,9\% construye sus ensayos con vocabulario académico, nominalizaciones y marcadores deónticos.

En este sentido, los perfiles de este estudio no solo entregan información sobre las estrategias discursivas utilizadas, sino también sobre los recursos léxico-gramaticales de lenguaje académico por los que optan los escritores en la producción de sus argumentaciones. Los resultados muestran que al interior de cada perfil de escritor se observan variaciones en el uso de los recursos del lenguaje académico. Dada la heterogeneidad de desempeños y la variedad de estrategias discursivas y lingüísticas utilizadas por los escritores en la producción de un género académico escolar, se puede señalar que no todos los estudiantes han tenido las mismas oportunidades para aprender a producir una argumentación. Como plantean Schleppegrell y Christie (2018), la construcción del texto y el desarrollo del escritor son procesos que van de la mano; en consecuencia, el análisis de los recursos de lenguaje académico ilumina sobre aquellos aspectos que hay que trabajar a nivel micro para poder avanzar en el dominio de las habilidades argumentativas. En este sentido, de particular interés resulta el perfil emergente para el grupo de estudiantes que presenta la mayor probabilidad de usar vocabulario temático; esto quiere decir, que utilizan en su ensayo palabras que estaban en el estímulo. Como señalan Phillips Galloway et al. (2020), los escritores que aún no han desarrollado sus habilidades de lenguaje utilizan esta estrategia para facilitar la tarea de producción dado su limitado repertorio lingüístico y se apoyan en las palabras entregadas en las consignas.

El bajo dominio de conocimiento y habilidades para argumentar de manera escrita se condice con el poco uso y despliegue que hacen los estudiantes de los recursos del lenguaje académico para poder argumentar. En consecuencia, es congruente pensar, como sugiere Larraín y Singer (2019), que no solo impacta en la competencia argumentativa el conocimiento, el valor retórico de las ideas, las instrucciones o las dificultades de la escritura, sino que también están en juego los recursos de lenguaje académico que los estudiantes puedan desplegar en sus argumentaciones escritas. En efecto, este estudio demuestra que, al interior de los distintos perfiles de escritores, se encuentran a su vez subgrupos según la probabilidad de usar distintos recursos de lenguaje académico lo que contribuye a descomponer las distintas demandas discursivas y lingüísticas involucradas en la producción de una argumentación. Así, los 
escritores emergentes se diferencian por la probabilidad de usar vocabulario temático o por usar nominalizaciones y vocabulario trandisciplinar. Asimismo, al interior del grupo de los escritores en desarrollo, quienes dominan un poco más la argumentación a nivel discursivo, se encuentran aquellos que tienen la probabilidad de usar los recursos de lenguaje académico de manera homogénea y aquellos que también despliegan recursos que predicen la calidad de la argumentación: nominalizaciones, vocabulario transdisciplinar y marcadores deónticos (Figueroa et al., en revisión).

En cuanto a las limitaciones de este estudio, se explora los perfiles de escritores y los recursos de lenguaje académico usados por estudiantes de $8^{\circ}$ básico que producen un ensayo de un tema de conocimiento general. Por lo tanto, las conclusiones son válidas para la argumentación escrita; más investigación se requiere con estudiantes de distintos niveles escolares y con otros géneros discursivos escolares. Asimismo, otros estudios podrían explorar el papel del conocimiento disciplinar en la calidad de las argumentaciones y cómo establecer un andamiaje y transferencia entre argumentaciones orales de conocimiento general, pasando por argumentaciones escritas para llegar a argumentaciones escritas disciplinares. Otra limitación importante de este estudio es que los perfiles de escritores se construyen solo para el ensayo sobre el uso del tablet; por lo tanto, es importante considerar que los recursos discursivos y lingüísticos no solo pueden variar entre géneros discursivos, sino también en la misma escritura argumentativa dependiendo de factores contextuales y temáticos lo que hace más dinámico el desempeño de la escritura argumentativa; por lo tanto, es fundamental considerar cuánto la interpretación de estos resultados puede variar si es que se realizan distintas tareas argumentativas en que varíen elementos contextuales.

Este estudio levanta información sobre la diversidad de perfiles de escritores a nivel discursivo y lingüístico al interior de un grupo, relevando la necesidad de fortalecer las herramientas de lenguaje de los estudiantes, mediante una interacción oral que favorezca la incorporación de los recursos de lenguaje académico de manera andamiada, así como implementar secuencias de enseñanza explícita de géneros discursivos escolares durante la educación primaria y a través de las distintas disciplinas. En efecto, intervenciones pedagógicas que se focalicen en el desarrollo de las ideas y el aprendizaje explícito de los recursos del lenguaje académico podrían favorecer un mejor desarrollo de argumentos que los estudiantes puedan desplegar para defender y potenciar sus puntos de vistas en sus textos.

Los resultados de este estudio sugieren que los estudiantes necesitan más oportunidades para aprender a construir argumentaciones durante la educación primaria, ya que esta actividad discursiva es un habilidad fundamental no solo para la construcción de aprendizajes a través de las disciplinas escolares (Kuhn et al., 
2016; Larraín \& Singer, 2019; Taylor et al., 2019), sino que también es clave para una participación ciudadana activa y crítica, cimiento indispensable para la democracia (Fuentes, 2019). Además, el uso del lenguaje académico en la argumentación es desafiante para los adolescentes, puesto que solo un grupo reducido de estudiantes despliega estrategias lingüísticas de este registro. Por otra parte, el estudio muestra que las dimensiones discursivas y lingüísticas se entrelazan y no necesariamente avanzan de la misma manera; por lo tanto, estrategias de enseñanza de la escritura diferenciada para distintos tipos de escritores necesitan ser implementadas para que los estudiantes puedan progresar en sus habilidades argumentativas escritas.

\section{Referencias}

Agencia de Calidad de la Educación (2015). Metodología de construcción de grupos socioeconómicos Simce 2013. URL= http://www.agenciaeducacion.cl/simce/documentos-tecnicos/

Akaike, Hirotugu. "Factor analysis and AIC”. Psychometrika 52 (1987): 317-332.

Álvarez, Gerardo. Textos y discursos: Introducción a la lingüística del texto. Concepción, Chile: Universidad de Concepción, 2001.

Andueza, Alejandra. "La escritura como herramienta de aprendizaje significativo: un cuasiexperimento en la clase de ciencias". Revista Complutense de Educación 27(2) (2016): 53-58.

Avalos, Mary, Margarita Gómez, Mileidis Gort and Walter Secada. "Hey! Today i will tell you about the water cycle! Variations of Language and Organizational Features in Third-Grade Science Explanation Writing". The elementary School Journal 118(1) (2017): 149-176.

Benítez, Ricardo y Marisol Velásquez. "Un análisis microestructural de la producción escrita de alumnos de $6^{\circ}$ y $8^{\circ}$ año de educación general básica”. Lenguas Modernas 26-27 (2000): 95-117.

Berman, Ruth. "Introduction: Developing discourse stance in different text types and languages". Journal of Pragmatics37 (2004): 105-124.

Berman, Ruth and Doris Ravid. "Becoming a literate language user. Oral and written text construction across adolescence”. En D. Olson, \& N. Torrance (Eds.), The Cambridge Handbook of Literacy (pp. 92-111). New York, NT: Cambridge University Press, 2009. 
Bronckart, Jean Paul. "Secuencias y otras formas de planificación”. En Jean Paul. Bronckart. Actividad verbal, textos y discursos. Por un interaccionismo sociodiscursivo (pp. 135-153). Madrid: Fundación Infancia y Aprendizaje, 2004.

Celeux, Gilles and Gilda Soromenho "An entropy criterion for assessing the number of clusters in a mixture model". Journal of Classification 13 (1996): 195-212.

Chamorro, Diana, Norma Barletta y Jorge Mizuno. "El lenguaje para enseñar y aprender las Ciencias Naturales: Un caso de oportunidades perdidas para la formación". Revista Signos 46(81) (2013): 3-28.

Chen, Hongling and Debra, Myhill. "Children talking about writing: Investigating metalinguistic understanding". Linguistics and Education, 35 (2016): 100-108.

Clogg, Cliford and Leo Goodman. "Latent structure analysis of a set of multidimensional contingency tables". Journal of the American Statistical Association 79(388) (1984): 762-771.

Concha, Soledad and Jean Paratore. "Local Coherence in Persuasive Writing: An Exploration of Chilean Students' Metalinguistic Knowledge, Writing Process, and Writing Products". Written Communication 28(1) (2011): 34-69.

Coxhead, Averil. “A new academic word list”. TESOL Quarterly 34(2) (2000): 213-238.

Crossley, Scott, Rod Roscoe and Danielle McNamara. "What is successful writing? An investigation into the multiple ways writers can write successful essays". Written Communication 31(2) (2014): 184-214.

Cummins, Jim. Bilingualism and minority-language children. Canadá, Toronto: OISE Press, 1981.

Emig, Jean. "Writing as a mode of learning". College Composition and Communication 28(2) (1977): 122-128.

Errázuriz, María Constanza. "Análisis del uso de los marcadores discursivos en argumentaciones escritas por estudiantes universitarios". Perfiles Educativos 34 (136) (2012): 98-117.

Figueroa, Javiera, Alejandra Meneses y Eugenio Chandía. "Desempeños en la calidad de explicaciones y argumentaciones en estudiantes chilenos de $8^{\circ}$ básico”. Revista Signos, 52(99) (2019): 31-54.

Figueroa, Javiera, Eugenio Chandía y Alejandra Meneses. "Calidad de la escritura en explicaciones y argumentaciones: validación estructural de una rúbrica para medir desempeños." Boletín de Lingüística, 30 (49-50) (2018): 43-67. 
Figueroa, Javiera, Alejandra Meneses y Eugenio Chandía. "Academic language and the quality of written arguments and explanations of Chilean 8th graders". Reading and Writing. 31(3) (2018): 703-723.

Friginal, Eric, Man Li and Sara Weigle. "Revisiting multiple profiles of learner compositions: A comparison of highly rated NS and NNS essays". Journal of Second Language Writing 23 (2014): 1-16.

Fuentes, Claudio. "La argumentación, entre pensamiento, democracia y aprendizaje". Cogency, Journal of Reasoning and Argumentation 11 (1-2) (2019). Editorial.

Galdames, Viviana, Lorena Medina, Ernesto San Martín, Rosa Gaete y Andrea Valdivia. “QQué actividades realizan los docentes de Nb1 para enseñar a leer en situación de evaluación docente? Enfoques tras las prácticas docentes”. En Jorge Manzi, Roberto González e Yulan Sun (Eds.), La evaluación docente en Chile (pp. 200-203). Santiago de Chile: MINEDUC/Ediciones Pontificia Universidad Católica de Chile, 2011.

García, Marta, Beatriz Hall y Marta Marín. "Ambigüedad, abstracción y polifonía del discurso académico: Interpretación de las nominalizaciones". Revista Signos 38(57) (2005): 49-60.

Glassner, Amnon and Baruch B. Schwarz. "The antilogos ability to evaluate information supporting arguments." Learning and Instruction 15 (4) (2005): 353-375.

Gómez, Gabriela, Carmen Sotomayor, Percy Bedwell, Ana María Domínguez and Elvira Jeldrez. "Analysis of lexical quality and its relation to writing quality for 4th grade, primary school students in Chile". Reading and Writing 29(7) (2016): 1317-1336.

Grøver, Vibeke, Paola Uccelli, Meredith Rowe and Elena Lieven. "Learning through language”. En Vibeke Grøver, Paola Uccelli, Meredith Rowe and Elena Lieven (Eds.). Learning through language. Towards an educationally informed theory of language learning, (pp. 1-16). Cambridge: Cambridge University Press, 2019.

Henríquez, Rodrigo, Andrés Carmona, Alen Quinteros y Mabelin Garrido. Leer y escribir para aprender historia: secuencias para la enseñanza y el aprendizaje del pensamiento histórico. Santiago: Ediciones UC, 2018.

Kuhn, Deanna, Laura Hemberger and Valerie Khait. "Dialogic argumentation as a bridge to argumentative thinking and writing”. Infancia y Aprendizaje 39(1) (2016): 25-48.

Larraín, Antonia y Vivian Singer. "Habilidades de argumentación de estudiantes de educación básica subvencionada en Chile y su relación con variables socio-educativas". Cogency, Journal of Reasoning and Argumentation 11 (1-2) (2019): 1-20. 
Larraín, Antonia, Christine Howe y Julieta Cerda. "Argumentation in Whole-Class Teaching and Science Learning". PSYKHE, 23 (2) (2014a): 1-15.

Larraín, Antonia, Paulina Freire y Trinidad Olivos. "Habilidades de argumentación escrita: Una propuesta de medición para estudiantes de quinto básico." Psicoperspectivas 13 (1) (2014b): 94-107.

MacWhinney, Brian. The CHILDES Project: Tools for analyzing talk. Electronic Edition. $\mathrm{URL}=\mathrm{http}: / /$ childes.psy.cmu.edu/, 2014.

McNamara, Danielle, Scott Crossley and Phillip McCarthy. "Linguistic features of writing quality”. Written Communication 27(1) (2010): 57-86.

Marinkovich, Juana. "Las estrategias cognitivo-retóricas y la dimensión dialéctica de la argumentación oral en una clase de lengua castellana y comunicación." Revista Signos 40 (63) (2007): 127-146.

Medina, Lorena, Andrea Valdivia, Rosa Gaete-Moscoso y Viviana Galdames. “¿Cómo enseñan a leer los profesores de $1^{\circ}$ y $2^{\circ}$ básico en un contexto de evaluación de desempeño docente en Chile?” Estudios Pedagógicos XLI (1) (2015): 183-198.

Meneses, Alejandra, Evelyn Hugo, Maximiliano Montenegro, Marcela Ruiz y Andrea Valenzuela. "Diferencias en recursos de lenguaje académico en explicaciones científicas producidas por estudiantes chilenos de $4^{\circ}$ grado". Boletín de lingüística 30 (49-50) (2018a): 134-157.

Meneses, Alejandra, Paola Uccelli, Verónica Santelices, Marcela Ruiz, Daniela Acevedo and Javiera Figueroa. "Academic language as a predictor of reading comprehension in monolingual Spanish-speaking readers: Evidence from Chilean early adolescents”. Reading Research Quarterly, 53(2) (2018b): 223-247.

Ministerio de Educación. Bases curriculares de $7^{\circ}$ básico a $2^{\circ}$ medio. Santiago: Unidad de Currículum y Evaluación. URL= https://curriculumnacional.mineduc.cl/614/articles-37136_bases.pdf

Miras, Mariana. "La escritura reflexiva. Aprender a escribir y aprender acerca de lo que se escribe". Reflective writing. Learning to write and learning about what you write. Infancia y aprendizaje 23(1) (2000): 65-80.

Montolío, Estrella. Conectores de la lengua escrita: contraargumentativos, consecutivos, aditivos y organizadores de la información. Barcelona: Ariel, 2001.

Myhill, Debra. "Children's patterns of composition and their reflections on their composing processes”. British Educational Research Journal 35 (1) (2009): 47-64. 
Myhill, Debra and Susan Jones. "Conceptualizing metalinguistic understanding in writing". Cultura y Educación 27(4) (2015): 839-867.

Nylund, Karn, Tihomir Asparouhov and Bengt Muthén. "Deciding on the number of classes in latent class analysis and growth mixture modeling: A Monte Carlo simulation study". Structural equation modeling: A multidisciplinary Journal 14(4) (2007): 535-569.

Olinghouse, Natalie and Joshua Wilson. "The relationship between vocabulary and writing quality in three genres". Reading and Writing 26(1) (2013): 45-65.

Parodi, Giovanni. "La evaluación de la producción de textos escritos argumentativos: una alternativa cognitivo/discursiva”. Revista Signos 33 (2000): 151-166.

Phillips Galloway, Emily, Juliane Stude and Paola Uccelli. “Adolescents' metalinguistic reflections on the academic register in speech and writing". Linguistics and Education 31 (2015): 221-237.

Phillips Galloway, Emily, Wenjuan Qin, Paola Uccelli, and Christopher Barr. "The role of cross-disciplinary academic language skills in disciplinary, source-based writing: investigating the role of core academic language skills in science summarization for middle grade writers". Reading and Writing 33 (2020): 13-44.

Poblete, Claudia. "Producción de textos argumentativos y metacognición". Letras 47 (2005): 63-88.

R Core Team. "A language and environment for statistical computing”. R Foundation for Statistical Computing, Vienna, Austria. 2017. URL= www.R-project.org/

Real Academia Española. Corpus del Español del Siglo XXI (CORPES). URL= http://web. frl.es/CORPES/org/publico/pages/consulta/entradaCompleja.view, 2016.

Rose, David and Martin, Jim R. Learning to write/learning to learn: Genre, knowledge and pedagogy in the Sydney school. London: Equinox, 2012.

Schwartz, Gideon. "Estimating the dimension of a model". The Annals of Statistics 6 (1978): 461-464.

Schleppegrell, Mary. The language of schooling. A functional linguistics perspective. Mahwah, NJ: Lawrence Erlbaum Publishers, 2004.

Schleppegrell, Mary and Francis Christie. "Linguistic features of writing development: A functional perspective". En Charles Bazerman, Arthur N. Applebee, Virginia W. Berninger, Deborah Brandt, Steve Graham, Jill V. Jeffery, Paul Kei Matsuda, Sandra Murphy, Deborah Wells Rowe, Mary Schleppegrell and Kristen Campbell Wil- 
132 - Cogency, Journal of reasoning and argumentation

cox (Eds.), The lifespan development of writing, (pp.111-150). Urbana, IL: National Council of Teacher of English, 2018.

Snow, Catherine and Paola Uccelli. “The challenge of academic language”. En David Olson \& Nancy Torrance (Eds.), The Cambridge Handbook of Literacy (pp. 112-133). New York: Cambridge University Press, 2009.

Solar, Horacio. "Implicaciones de la argumentación en el aula de matemáticas". Revista Colombiana de Educación, (74),(2018): 155-176.

Sotomayor, Carmen, Gabriela Gómez, Elvira Jéldrez, Percy Bedwell, Ana María Domínguez y Natalia Ávila. "Evaluación analítica de la escritura de estudiantes de $4^{\circ}$ año básico en Chile. Onomázein 34 (2016): 381-425.

Taylor, Karen S., Joshua F., Lawrence, Carol M. Connor, Catherine E. Snow. "Cognitive and linguistic features of adolescent argumentative writing: Do connectives signal more complex reasoning?" Reading and Writing 32 (2019): 983-1007.

Uccelli, Paola. "Learning the language for school literacy”. En Vibeke Grøver, Paola Uccelli, Meredith Rowe and Elena Lieven (Eds.), Learning through language. Towards an educationally informed theory of language learning, (pp. 95-109). Cambridge: Cambridge University Press, 2019.

Uccelli, Paola, Christopher Barr, Christina Dobbs, Emily Phillips Galloway, Alejandra Meneses and Emilio Sánchez. "Core academic language skills: An expanded operational construct and a novel instrument to chart school-relevant language proficiency in preadolescent and adolescent learners". Applied Psycholinguistics 36(5) (2015): 1077-1109.1-33.

Uccelli, Paola, Christina Dobbs and Jessica Scott. "Mastering academic language: organization and stance in the persuasive writing of high school students". Written Communication 30(1) (2013): 36-62.

Uccelli, Paola, Emily Phillips Galloway, Christopher Barr, Alejandra Meneses, and Christina Dobbs. "Beyond vocabulary: Exploring cross-disciplinary academic-language proficiency and its association with reading comprehension". Reading Research Quarterly 50(3) (2015): 337-356.

Uccelli, Paola y Alejandra Meneses. "Habilidades de lenguaje académico y su asociación con la comprensión de lectura en la escuela primaria y media: un nuevo constructo operacional". Miríada Hispánica 10 (2015): 179-206.

Valenzuela, Juan Pablo, Cristian Bellei \& Danae De Los Ríos. "Socioeconomic school segregation in a market-oriented educational system. The case of Chile". Journal of Education Policy 29(2) (2014): 217-241. 
Villarroel, Constanza, Mercè Garcia-Mila, Mark Felton and Andrea Miralda- Banda. "Effect of argumentative goals in the quality of argumentative dialogue and written argumentation". Infancia y Aprendizaje (2018): 1-21.

Waes, Luuk and Peter Schellens. "Writing profiles: the effect of the writing mode on pausing and revision patterns of experienced writers". Journal of Pragmatics 35 (2003): 829-853.

Zavala, Virginia. "La escritura académica y la agencia de los sujetos". Cuadernos Comillas 1 (1) (2011): 52-66. 